\title{
Surface photometry of early-type galaxies in the Hubble deep field ${ }^{\star}$
}

\author{
G. Fasano ${ }^{1}$ and M. Filippi ${ }^{2}$ \\ 1 Astronomical Observatory of Padova, Vicolo dell' Osservatorio 5, 35122 Padova, Italy \\ 2 Department of Astronomy of the Padova University, Vicolo dell' Osservatorio 5, 35122 Padova, Italy \\ e-mail gfasano@leda.pd.astro.it
}

Received September 16; accepted November 5, 1997

\begin{abstract}
The detailed surface photometry of a sample of early-type galaxies in the Hubble Deep Field is presented as part of a long-term project aimed to settle strong observational constraints to the theories modelling the evolution of elliptical galaxies from the early stages.

The sample has been extracted, in the $V_{606}$ band, from the database provided by the ESO-STECF-HDF Group (Couch 1996). The selection criteria involve the total magnitude, the number of pixels detected above the background level and an automatic star/galaxy classifier. Moreover, form visual inspection of the frames, we excluded the galaxies showing unambiguous late-type morphology. The analysis of the luminosity and geometrical profiles, carried out on the 162 candidates obeying our selection criteria, resulted in a list of 99 "bona fide" earlytype galaxies, for which accurate total magnitudes and effective radii were computed on the basis of the equivalent luminosity profiles. The comparison with the magnitudes given by Williams et al. (1996) indicates that the automated photometry tends to underestimate the total luminosity of the ellipticals.
\end{abstract}

The luminosity profiles of most of galaxies in our sample follow fairly well the de Vaucouleurs law ("Normal" profiles). However, a relevant fraction of galaxies, even following the $r^{1 / 4}$ law in the main body light distribution, exhibit in the inner region a flattening of the luminosity profile not attributable to the PSF ("Flat" profiles) or, in some cases, a complex (multi-nucleus) structure ("Merger" profiles). A statistically significant correlation is found between the shapes of the luminosity profiles and the ellipticity distribution. In particular, the average ellipticity of galaxies belonging to the "Flat" and "Merger" classes is significantly higher than that of the "Normal" galaxies. Finally, even taken into account the relevant un-

Send offprint requests to: G. Fasano

* Complete Table 1 is available electronically at the CDS at ftp 130.79.128.5 and Fig. 1 is can be accessed on the online version of the journal (http://www.edpsciences.com). certainty of the outer position angle profiles, the amount of isophotal twisting of HDF ellipticals turns out to be significantly larger with respect to that of the local samples.

Key words: galaxies: elliptical and lenticular — galaxies: fundamental parameters - galaxies: photometry

\section{Introduction}

Understanding the processes governing the formation and the evolution of galaxies from the early stages is one of the major goals of the present day cosmology. Comprehensive numerical codes, linked with powerful CPU capabilities, make now possible to follow the evolution of primordial density fluctuations down to the stages preceding the formation of real galaxies (see Jenkins et al. 1997 for a recent review). White (1997) has also shown that the scaling relations expected after evolution of proto-galaxies of different morphologies seem to be in good agreement with those observed today, i.e. the Tully-Fisher (1977) and the Fundamental Plane (Dressler et al. 1987; Djorgovski \& Davis 1987) relations.

Still, a number of key questions remain to be answered. We mention (among the others) the following ones: which is the parameter driving galaxies towards different morphologies? Are elliptical galaxies originated from gravitational collapse of primordial fluctuations (single burst of star formation whose duration depends on the onset of galactic winds) or are they the result of multiple merging (infall and recursive bursts)? Which is the influence of the internal and intergalactic absoption in determining the observed brightness and color profiles of high redshift galaxies?

Besides the global approach to the star formation history (Madau et al. 1997), answering these questions also requires detailed morphological and dynamical studies of 
galaxies in the early evolutionary stages. In particular, luminosity and geometrical profiles in different bands are needed to obtain unambiguous morphological classifications as well as reliable estimates of the galaxy sizes at intermediate and high redshifts.

Recent advances in the sensitivity and resolution of the observations both in imaging and spectroscopy with the Hubble Space Telescope (HST) and from the ground have greatly enlarged the horizon of morphological and dynamical studies for high redshift galaxies (Giavalisco et al. 1996; van Dokkum \& Franx 1996; Shade et al. 1996; Shade et al. 1997; Oemler et al. 1997; Lowenthal et al. 1997; Pettini et al. 1997 and references therein). The Hubble Deep Field (HDF) is perhaps the most impressive example of these progresses (Ellis 1997).

The HDF project (Williams et al. 1996) has been realized using observational procedures (dithering) and data handling techniques (drizzling) aimed to improve not only the cosmetic of the final images, but also the resolution performances. In particular, after "drizzling", the pixel size in arcseconds of the three WF cameras turns out to be even better than that of the PC ( $\sim$ "'.040 vs. $\sim$ "'046). Moreover, the very long total exposure times of the final images allow to overcome the main limitation of the WFPC data, that is the relatively high surface brightness level usually reached for extended sources. This makes the HDF frames particularly suited in order to perform the detailed surface photometry of objects whose surface luminosity slowly decreases outwards down to very faint levels, as in the case of elliptical galaxies. We can rightfully assert that the HDF represents the best opportunity we had since now to study the morphology of elliptical galaxies at very high redshifts.

We have undertaken a long-term project aimed to produce the detailed surface photometry, in different bands, for a sample of early-type galaxies in the HDF. The main scientific goal of this project is to settle strong observational constraints to the theories modelling the evolution of elliptical galaxies from the early stages (e.g. Tantalo et al. 1996; Kauffmann \& Charlot 1998; Chiosi et al. 1997 and references therein).

In this paper we present the surface photometry in the $V_{606}$ band. In the forthcoming papers (Filippi \& Fasano 1998; Fasano et al. 1998) we will present the surface photometry in the remaining 3 bands and discuss the improvements that the morphological information, together with the photometry in different optical and infrared bands, can produce in understanding the processes of galaxy formation and evolution.

In Sect. 2 we discuss the sample selection. Section 3 illustrates the techniques we used to extract the morphological information from the HDF frames. In Sect. 4 we present the results of the detailed surface photometry and discuss the global morphological properties of the sample.

\section{Sample selection}

Our sample of early-type galaxies has been extracted from the second release of the WFPC2-HDF frames, in the $V_{606}$ band. Due to the efficiency curve of the WFPC2, this filter usually ensures a better $\mathrm{S} / \mathrm{N}$ ratio with respect to the other available filters $\left(U_{300}, B_{450}\right.$ and $\left.I_{814}\right)$ even in the case of high redshift galaxies, as we verified by comparing the $V_{606}$ and $I_{814}$ images.

\subsection{Selection criteria}

The sample selection is based on the compilation provided by the ESO-STECF-HDF Group, obtained through the automated SExtractor algorithm (Bertin \& Arnouts 1996). Among the other things, the catalog includes, for each object in the field, the total $V_{606}$ magnitude in the STMAG system, the number of pixels $\left(N_{\text {pix }}\right)$ detected above the background using a threshold of $1.3 \sigma$ of the noise, and a star/galaxy $(s / g)$ classifier, ranging from 0 to 1 , which gives the probability that the object is stellar $(s / g=1$ means "star"). After a preliminar inspection of the frames, supplemented by several fast average-tracing luminosity profiles of faint and/or small objects, we decided to set the following limits for inclusion in our preliminar sample: (1) $V_{606}$ (STMAG) $\leq 26.5 ;$ (2) $N_{\text {pix }} \geq$ $200 ;(3)(s / g) \leq 0.6$.

As for the first two limits, they concern essentially the technical "feasibility" of the morphological analysis. Although at this stage we preferred not to be too much severe in the selection, these limits provide completeness criteria to our sample. The choice of the third limit turned out not to be critical, probably due to the very shape of the star profiles. Nevertheless, in this case we preferred to be more conservative, in order to prevent the inclusion of stars in our sample.

\subsection{Morphological screening}

We found 372 objects of the SExtractor catalog matching the above limits in the three chips of the WFC (the catalog does not include the PC). 29 objects from the chip \#1 (PC) were added later to this list by simple visual inspection, so that the total preliminar sample turned out to be of 401 objects.

We used the "imexam" IRAF's tool, together with the "Saoimage" display facility, to estimate the morphological type of each object, thus producing a first screening of the galaxies in the sample. Apart from some cases, the angular resolution did not allow us to give precise Hubble types. Moreover, many faint galaxies appear heavily disturbed by the presence of close (often multiple) companions or peculiar structures, which obviously make even more difficult the classification. Nevertheless, most of the "early-type" candidates turned out to be easily recognizable on the basis of the light concentration. The doubious cases (mainly 
$S 0 s$ and $S a$ galaxies) were retained at this stage and were examined later in a quantitative way (see next section).

After this selection we were left with 134 galaxies. In order to check the reliability of our classifications, we compared them with those given by Van den Bergh et al. (1996) and by Statler (1996, private communication). Due to the different selection criteria, the three samples do not overlap exactly each other. Nevertheless, for the common objects the agreement was fairly good ( $80 \%$ with Van den Bergh et al. and $84 \%$ with Statler). To be more accurate, we decided to provisionally include in our sample all galaxies matching our selection criteria which, contrary to our estimates, were classified as "early-type" by Van den Bergh et al. (1996) and/or by Statler (1996). In this way 28 more galaxies enriched our sample, which become of 162 objects.

\subsection{Final sample}

We performed the detailed surface photometry of this sample, producing luminosity and geometrical profiles of each object. The next Section outlines the techniques we used to extract the morphological information from this very peculiar observational material. From the analysis of the luminosity and geometrical profiles, 34 objects of the sample have been recognized to be "disk-dominated" objects (likely $S a$ galaxies), whereas 28 more objects showed peculiar or unclassifiable profiles. These galaxies were excluded from the final sample of "bona fide" early-type galaxies.

The final sample of 99 galaxies is reported in Table 1, where Col. (1) gives the FOCAS list identification (Williams et al. 1996), Cols. (2) and (3) the J2000 coordinates $\alpha$ and $\delta$, Col. (4) our morphological classification, Cols. (5) the morphological type (if available) given by van den Bergh et al. (1996).

\section{Detailed surface photometry}

The detailed surface photometry of our sample of HDF ellipticals in the $V_{606}$ band was performed using the AIAP package (Fasano 1990), which is equipped with a completely interactive graphical interface, allowing flexible tools for sky subtraction, drawing, masking and fitting of the isophotes, PSF evaluation, etc. These capabilities, as well as the allowance for checking "on-line" all steps of the procedure, turn out to be particularly useful when handling morphologically complex structures and/or closely interacting objects, a situation quite common in the case of the HDF galaxies.

Our magnitudes are given in the $S T M A G$ system.

\subsection{Basic procedures}

The second release of the HDF provided us with frames "ready to be used", in the sense that all the standard reduction procedures (flat fielding, bias and dark-current removal, etc.), as well as the specific ones (drizzling, position weighting, etc...), were already performed and the proper (constant) sky background was subtracted from each frame.

Nevertheless, since not negligible and systematic residuals in the backgrounds were detected, we decided to perform for each galaxy a "local" refinement of the background subtraction by linear interpolation of the residuals. After this refinement the mean (systematic) background variations were estimated to be of the order of $\sim 0.5 \%$ of the original sky levels. These systematic variations have been used to estimate error bars in the outer luminosity and geometrical profiles of each object (see next subsection).

The isophotes were drawn with a fixed surface brightness step of 0.2 . Therefore, at least in the inner part of galaxies having steep luminosity profiles, isophotes might be oversampled, since the difference of radius between two successive isophotes might be lower than the pixelsize. We note, however, that the shapes of luminosity profiles are not modified by the oversampling and that, whenever we introduce luminosity profiles weighting (i.e. fitting with analytical functions and extrapolation), we set to zero the weight of oversampled isophotes.

Low surface brightness isophotes (usually $\mu_{606} \geq$ $25.8)$ were drawn after rebinning of the frames. The AIAP package allows to decide interactively when and how perform the rebinning, depending on the noise of each isophote. The maximum reachable surface brightness was not the same for all galaxies. It essentially turned out to depend on the presence of close companions and/or irregular sub-structures which often make useless to go much deep in drawing the isophotes. We can reach easily the surface brightness level of $28^{\mathrm{m}} 0$. In the most favourable cases we were able to reach $\mu_{606}=28$. 8 .

The isophotes of HDF ellipticals are often quite irregular due to the intrinsic complexity of the structures (which is likely to increase at increasing redshift), as well as to the presence of close (possibly interacting) companions. Therefore, the flexibility of the AIAP masking and ellipse fitting tools turned out to be useful in order to secure reliable profiles even for very intriguing cases. We mention, among the other things, the possibility to take fixed or relaxed (for each isophote) the coordinates of the center of the fitting ellipses and the possibility to complete the masked parts of the isophotes by using the corresponding symmetric parts of the same isophotes with respect to the center.

\subsection{Extraction of profiles and error estimates}

Once the isophotes have been carefully masked and interpolated through ellipses, we obtained luminosity and geometrical profiles of all galaxies in our sample. In particular, we obtained surface brightness, ellipticity, position angle and coefficients of the Fourier analysis of the 
Table 1. The sample

\begin{tabular}{|c|c|c|c|c|c|c|c|c|c|c|c|c|}
\hline \multirow[t]{2}{*}{ FOCAS-ID } & \multicolumn{4}{|c|}{ Coordinates (2000) } & \multicolumn{2}{|c|}{ Morphology } & \multirow{2}{*}{$\begin{array}{l}\text { Lum.Prof. } \\
\text { class }\end{array}$} & \multirow{2}{*}{$\begin{array}{l}V_{606}^{\mathrm{Tot}}\left(\Delta_{m}\right) \\
\text { (STMAG) }\end{array}$} & \multirow{2}{*}{$\begin{array}{l}r_{\mathrm{e}}^{\mathrm{eq}} \\
\left({ }^{\prime \prime}\right)\end{array}$} & \multirow[t]{2}{*}{$\varepsilon_{\mathrm{e}}$} & \multirow[t]{2}{*}{$\varepsilon_{\max }$} & \multirow{2}{*}{$\begin{array}{l}\Delta \theta \\
\left({ }^{\circ}\right)\end{array}$} \\
\hline & & & & & & & & & & & & \\
\hline ID_4_942_0 & $36^{m}$ & ${ }^{n} 39^{s} .43$ & $12^{\prime}$ & $11^{\prime \prime} .76$ & $\mathrm{E}$ & $\mathrm{E}$ & 1 & $24.75(.33)$ & 0.54 & 0.18 & 0.25 & 15 \\
\hline ID_4_926_2 & 36 & 39.56 & 121 & 13.83 & $\mathrm{~m}$ & $\mathrm{p}$ & 3 & $25.36(.17)$ & 0.19 & 0.04 & 0.17 & 60 \\
\hline ID_4_928_1 & 36 & 40.01 & 12 & 07.37 & So & $\mathrm{E}$ & 1 & $23.61(.15)$ & 0.36 & 0.10 & 0.19 & 50 \\
\hline ID_4_878_2 & 36 & 40.74 & 12 & 04.96 & $\mathrm{E}$ & $\mathrm{E}$ & 2 & $25.79(.19)$ & 0.22 & 0.35 & 0.35 & 8 \\
\hline ID_4_878_11 & 36 & 40.96 & 12 & 05.31 & $\mathrm{E}$ & Sap & 2 & $23.66(.14)$ & 0.22 & 0.12 & 0.14 & 30 \\
\hline ID_4_822_0 & 36 & 41.15 & 121 & 10.56 & $\mathrm{E}$ & $\mathrm{E}$ & 1 & $25.42(.27)$ & 0.33 & 0.08 & 0.10 & 5 \\
\hline ID_4_858_13 & 36 & 41.25 & 12 & 03.07 & $\mathrm{E}$ & $\mathrm{E}$ & 1 & $25.18(.23)$ & 0.15 & 0.14 & 0.19 & 11 \\
\hline ID_4_767_0 & 36 & 41.49 & 12 & 14.98 & So & $\mathrm{E}$ & 2 & $25.16(.15)$ & 0.19 & 0.36 & 0.39 & 6 \\
\hline ID_4_661_1 & 36 & 41.62 & 123 & 35.67 & $\mathrm{E}$ & $\mathrm{E}$ & 2 & $24.96(.17)$ & 0.22 & 0.18 & 0.23 & 20 \\
\hline ID_4_639_1 & 36 & 41.71 & 123 & 38.75 & $\mathrm{E}$ & Et? & 1 & $25.12(.14)$ & 0.17 & 0.08 & 0.16 & 47 \\
\hline ID_4_937_0 & 36 & 42.28 & 112 & 26.18 & $\mathrm{E}$ & $\mathrm{E}$ & 1 & $25.50(.19)$ & 0.18 & 0.19 & 0.22 & 30 \\
\hline ID_1_95_0 & 36 & 42.38 & 13 & 19.36 & $\mathrm{E}$ & - & 1 & 25.03(.13) & 0.13 & 0.28 & 0.30 & 12 \\
\hline ID_4_602_0 & 36 & 42.41 & 123 & 32.48 & $\mathrm{E}$ & $\mathrm{E}$ & 1 & $25.30(.18)$ & 0.16 & 0.24 & 0.28 & 15 \\
\hline ID_4_804_0 & 36 & 42.53 & 115 & 50.01 & $\mathrm{E}$ & Ep? & 1 & $25.40(.16)$ & 0.20 & 0.23 & 0.27 & 8 \\
\hline ID_4_774_3 & 36 & 42.78 & 115 & 54.28 & $\mathrm{E}$ & - & 1 & 25.95(.18) & 0.14 & 0.20 & 0.21 & 26 \\
\hline ID_4_581_11 & 36 & 42.87 & 12 & 27.85 & $\mathrm{E} / \mathrm{S} 0$ & - & 1 & $25.77(.15)$ & 0.14 & 0.19 & 0.25 & 40 \\
\hline ID_4_845_0 & 36 & 42.92 & 113 & 37.27 & $\mathrm{E}$ & $\mathrm{Sa}$ & 2 & $25.08(.16)$ & 0.20 & 0.37 & 0.37 & 3 \\
\hline ID_4_554_1 & 36 & 43.13 & 122 & 28.11 & Ep & Ep & 2 & $25.58(.20)$ & 0.17 & 0.29 & 0.33 & 38 \\
\hline ID_4_493_0 & 36 & 43.16 & 124 & 42.20 & So & $\mathrm{E}$ & 1 & $23.26(.24)$ & 0.44 & 0.33 & 0.33 & 7 \\
\hline ID_4_727_0 & 36 & 43.41 & 115 & 51.57 & $\mathrm{E}$ & Ep? & 2 & $23.43(.10)$ & 0.21 & 0.49 & 0.48 & 3 \\
\hline ID_4_565_0 & 36 & 43.63 & 121 & 18.25 & $\mathrm{~m}$ & Sap & 3 & $23.46(.12)$ & 0.31 & 0.18 & 0.28 & 65 \\
\hline ID_4_744_0 & 36 & 43.80 & 114 & 42.88 & $\mathrm{E}$ & - & 1 & 22.33(.18) & 0.63 & 0.12 & 0.16 & 50 \\
\hline ID_2_82_1 & 36 & 44.07 & $14 \mathrm{C}$ & 09.92 & $\mathrm{E}$ & $\mathrm{E} / \mathrm{m}$ & 2 & $24.92(.15)$ & 0.18 & 0.27 & 0.28 & 19 \\
\hline ID_4_752_1 & 36 & 44.38 & 113 & 33.20 & $\mathrm{E}$ & - & 1 & $22.97(.22)$ & 0.90 & 0.18 & 0.18 & 3 \\
\hline ID_1_56_0 & 36 & 44.46 & 13 & 13.10 & $\mathrm{E}$ & - & 1 & 26.38(.19) & 0.13 & 0.15 & 0.21 & 7 \\
\hline ID_4_579_0 & 36 & 44.74 & 115 & 57.05 & $\mathrm{E}$ & $\mathrm{E} / *$ & 1 & $25.53(.14)$ & 0.13 & 0.06 & 0.09 & 93 \\
\hline ID_1_37_1 & 36 & 44.79 & 13 & 07.23 & $\mathrm{~m}$ & - & 3 & $25.75(.18)$ & 0.13 & 0.50 & 0.46 & 28 \\
\hline ID_2_163_0 & 36 & 45.29 & $14 \mathrm{C}$ & 07.03 & $\mathrm{E}$ & - & 1 & 25.94(.19) & 0.18 & 0.25 & 0.26 & 15 \\
\hline ID_4_555_2 & 36 & 45.33 & 115 & 54.52 & $\mathrm{E} / \mathrm{S} 0$ & $\mathrm{E}$ & 1 & $24.79(.21)$ & 0.32 & 0.17 & 0.24 & 20 \\
\hline ID_4_368_0 & 36 & 45.35 & 123 & 33.70 & $\mathrm{E} / \mathrm{S} 0$ & - & 1 & $26.07(.22)$ & 0.24 & 0.26 & 0.25 & 25 \\
\hline ID_2_80_0 & 36 & 45.40 & 135 & 50.07 & $\mathrm{E}$ & - & 2 & $25.64(.18)$ & 0.18 & 0.26 & 0.28 & 8 \\
\hline ID_1_100_0 & 36 & 45.52 & 132 & 29.97 & $\mathrm{E}$ & - & 1 & $26.37(.14)$ & 0.13 & 0.12 & 0.29 & 22 \\
\hline ID_1_35_0 & 36 & 45.61 & 13 & 08.92 & $\mathrm{E}$ & - & 1 & $24.35(.16)$ & 0.21 & 0.24 & 0.26 & 14 \\
\hline ID_4_516_0 & 36 & 45.65 & 115 & 53.97 & $\mathrm{E}$ & Et & 1 & $25.30(.15)$ & 0.18 & 0.13 & 0.20 & 40 \\
\hline ID_4_497_0 & 36 & 45.73 & 115 & 57.31 & $\mathrm{E}$ & - & 1 & $26.05(.17)$ & 0.13 & 0.12 & 0.14 & 20 \\
\hline ID_4_520_0 & 36 & 45.79 & 115 & 50.52 & $\mathrm{E} / \mathrm{S} 0$ & - & 1 & $26.07(.19)$ & 0.14 & 0.11 & 0.18 & 15 \\
\hline ID_2_61_0 & 36 & 46.12 & 133 & 34.62 & $\mathrm{E}$ & $\mathrm{E}$ & 2 & $25.91(.15)$ & 0.15 & 0.26 & 0.31 & 10 \\
\hline ID_4_254_0 & 36 & 46.13 & 124 & 46.50 & $\mathrm{E}$ & $\mathrm{E} / *$ & 1 & $23.64(.19)$ & 0.55 & 0.27 & 0.17 & 45 \\
\hline ID_1_47_0 & 36 & 46.16 & 13 & 13.89 & $\mathrm{E}$ & - & 2 & $25.17(.13)$ & 0.15 & 0.38 & 0.37 & 16 \\
\hline ID_4_322_2 & 36 & 46.21 & 122 & 28.43 & $\mathrm{E}$ & Et & 2 & $25.59(.16)$ & 0.16 & 0.23 & 0.28 & 5 \\
\hline ID_2_251_0 & 36 & 46.34 & $14 \mathrm{C}$ & 04.62 & $\mathrm{E} / \mathrm{S} 0$ & - & 1 & $22.96(.17)$ & 0.52 & 0.05 & 0.14 & 110 \\
\hline ID_4_471_0 & 36 & 46.51 & 115 & 51.32 & $\mathrm{E}$ & $\mathrm{E}$ & 1 & $23.11(.07)$ & 0.22 & 0.08 & 0.12 & 45 \\
\hline ID_4_289_0 & 36 & 46.95 & 122 & 26.08 & $\mathrm{E}$ & $\mathrm{E}$ & 1 & $25.73(.14)$ & 0.16 & 0.11 & 0.20 & 49 \\
\hline ID_2_201_0 & 36 & 47.18 & 134 & 41.82 & $\mathrm{E}$ & Et & 1 & $24.45(.16)$ & 0.14 & 0.15 & 0.24 & 15 \\
\hline ID_2_272_0 & 36 & 47.68 & 135 & 51.28 & $\mathrm{E}$ & - & 2 & $25.98(.16)$ & 0.17 & 0.26 & 0.28 & 34 \\
\hline ID_2_363_0 & 36 & 47.71 & $14 \mathrm{C}$ & 09.43 & $\mathrm{E}$ & - & 2 & $25.94(.15)$ & 0.15 & 0.25 & 0.33 & 17 \\
\hline ID_2_121_111 & 36 & 48.08 & 13 & 09.02 & S0 & - & 1 & $21.48(.11)$ & 0.60 & 0.04 & 0.23 & 30 \\
\hline ID_2_412_11 & 36 & 48.11 & 141 & 14.42 & $\mathrm{E} / \mathrm{S} 0$ & Sap & 1 & $25.42(.22)$ & 0.17 & 0.27 & 0.42 & 7 \\
\hline ID_4_260_112 & 36 & 48.12 & 121 & 14.90 & $\mathrm{E}$ & $\mathrm{E}$ & 2 & $25.08(.13)$ & 0.18 & 0.30 & 0.28 & 13 \\
\hline ID_2_449_1 & 36 & 48.34 & 141 & 16.63 & $\mathrm{E}$ & $\mathrm{E}$ & 2 & $24.11(.22)$ & 0.22 & 0.24 & 0.30 & 15 \\
\hline ID_2_173_0 & 36 & 48.47 & 13 & 16.62 & $\mathrm{E}$ & $\mathrm{E}$ & 2 & $23.74(.29)$ & 0.72 & 0.18 & 0.35 & 8 \\
\hline ID_2_537_12 & 36 & 48.71 & 142 & 22.62 & $\mathrm{E}$ & Et & 2 & $25.60(.15)$ & 0.20 & 0.32 & 0.35 & 12 \\
\hline ID_3_51_0 & 36 & 48.72 & 13 & 02.45 & $\mathrm{E}$ & $\mathrm{E} / *$ & 1 & $25.93(.17)$ & 0.17 & 0.08 & 0.14 & 5 \\
\hline ID_2_236_2 & 36 & 48.97 & 132 & 21.88 & $\mathrm{E}$ & Et & 1 & $24.81(.10)$ & 0.16 & 0.03 & 0.13 & 65 \\
\hline ID_2_180_0 & 36 & 49.05 & 13 & 09.64 & $\mathrm{E}$ & $\mathrm{Sa}$ & 2 & $24.65(.15)$ & 0.20 & 0.50 & 0.47 & 4 \\
\hline ID_4_274_0 & 36 & 49.11 & 115 & 50.54 & So & - & 2 & $26.02(.25)$ & 0.17 & 0.23 & 0.24 & 10 \\
\hline ID_2_264_2 & 36 & 49.38 & 131 & 11.22 & $\mathrm{E}$ & $\mathrm{E}$ & 1 & $22.80(.11)$ & 0.21 & 0.24 & 0.26 & 30 \\
\hline ID_2_456_1111 & 36 & 49.44 & 13 & 46.88 & S0 & - & 1 & $18.93(.05)$ & 0.52 & 0.37 & 0.39 & 7 \\
\hline ID_3_229_0 & 36 & 49.48 & 124 & 48.73 & $\mathrm{E}$ & $\mathrm{E} / *$ & 2 & $24.96(.24)$ & 0.24 & 0.10 & 0.12 & 15 \\
\hline ID_2_590_1 & 36 & 49.51 & 142 & 21.10 & $\mathrm{E}$ & - & 2 & $25.89(.21)$ & 0.16 & 0.18 & 0.16 & 55 \\
\hline ID_4_109_0 & 36 & 49.60 & 121 & 12.68 & $\mathrm{E}$ & $\mathrm{p}$ & 2 & $25.29(.12)$ & 0.16 & 0.38 & 0.40 & 10 \\
\hline ID_3_143_0 & 36 & 49.64 & 125 & 57.43 & $\mathrm{~m}$ & Sap & 3 & $22.85(.15)$ & 0.32 & 0.55 & 0.65 & 4 \\
\hline
\end{tabular}


Table 1. continued

\begin{tabular}{|c|c|c|c|c|c|c|c|c|c|c|c|c|}
\hline \multirow{3}{*}{$\begin{array}{l}\text { FOCAS-ID } \\
\text { ID_3_243_0 }\end{array}$} & \multicolumn{4}{|c|}{ Coordinates (2000) } & \multicolumn{2}{|c|}{ Morphology } & \multirow{2}{*}{$\begin{array}{l}\text { Lum.Prof. } \\
\text { class }\end{array}$} & \multirow{2}{*}{$\begin{array}{l}V_{606}^{\operatorname{Tot}}\left(\Delta_{m}\right) \\
\text { (STMAG) }\end{array}$} & \multirow{2}{*}{$\begin{array}{l}r_{\mathrm{e}}^{\mathrm{eq}} \\
\left({ }^{\prime \prime}\right)\end{array}$} & \multirow[t]{2}{*}{$\varepsilon_{\mathrm{e}}$} & \multirow[t]{2}{*}{$\varepsilon_{\max }$} & \multirow{2}{*}{$\begin{array}{l}\Delta \theta \\
\left(^{\circ}\right)\end{array}$} \\
\hline & & & & & Our & V.d.Bergh & & & & & & \\
\hline & 36 & 49.81 & 12 & 48.79 & E/S0p & Ep & 1 & $25.52(.21)$ & 0.21 & 0.32 & 0.34 & 14 \\
\hline ID_2_456_22 & 36 & 50.03 & 13 & 51.99 & S0 & Ep & 1 & $25.15(.12)$ & 0.17 & 0.36 & 0.50 & 6 \\
\hline ID_3_321_1 & 36 & 50.27 & 12 & 45.75 & So & - & 1 & $23.05(.13)$ & 0.43 & 0.34 & 0.36 & 5 \\
\hline ID_2_373_0 & 36 & 50.30 & 13 & 29.73 & $\mathrm{E}$ & $\mathrm{E}$ & 1 & $25.58(.18)$ & 0.16 & 0.27 & 0.33 & 9 \\
\hline ID_2_725_0 & 36 & 50.56 & 14 & 28.47 & $\mathrm{E}$ & $\mathrm{E}$ & 2 & $25.50(.15)$ & 0.17 & 0.52 & 0.44 & 12 \\
\hline ID_2_693_1 & 36 & 51.35 & 14 & 11.02 & $\mathrm{E}$ & $\mathrm{E}$ & 2 & $25.22(.15)$ & 0.18 & 0.18 & 0.25 & 22 \\
\hline ID_3_659_2 & 36 & 51.44 & 12 & 20.71 & Ep & $\mathrm{E} / \mathrm{S} 0$ & 1 & $25.69(.15)$ & 0.13 & 0.18 & 0.17 & 23 \\
\hline ID_3_902_1 & 36 & 51.79 & 11 & 57.81 & $\mathrm{E} / \mathrm{Sa}$ & - & 2 & $26.25(.22)$ & 0.16 & 0.18 & 0.20 & 42 \\
\hline ID_2_531_0 & 36 & 51.97 & 13 & 32.18 & $\mathrm{E}$ & $\mathrm{E}$ & 3 & $24.06(.15)$ & 0.29 & 0.10 & 0.07 & 15 \\
\hline ID_3_586_0 & 36 & 52.10 & 12 & 26.31 & $\mathrm{E}$ & $\mathrm{E} / \mathrm{Sa}$ & 1 & $25.77(.36)$ & 0.36 & 0.14 & 0.19 & 20 \\
\hline ID_2_646_0 & 36 & 52.23 & 13 & 48.07 & $\mathrm{E}$ & $\mathrm{E}$ & 2 & $25.14(.21)$ & 0.20 & 0.42 & 0.38 & 5 \\
\hline ID_2_849_0 & 36 & 52.40 & 14 & 20.95 & $\mathrm{E}$ & Ep & 2 & $25.60(.26)$ & 0.19 & 0.32 & 0.30 & 15 \\
\hline ID_3_625_0 & 36 & 52.51 & 12 & 24.78 & $\mathrm{~m}$ & $\mathrm{p}$ & 3 & $25.39(.13)$ & 0.15 & 0.50 & 0.49 & 3 \\
\hline ID_3_696_0 & 36 & 52.69 & 12 & 19.72 & $\mathrm{E}$ & $\mathrm{E}$ & 2 & $23.77(.09)$ & 0.19 & 0.46 & 0.45 & 10 \\
\hline ID_2_637_0 & 36 & 52.76 & 13 & 39.08 & $\mathrm{E}$ & $\mathrm{E} / *$ & 1 & $25.18(.15)$ & 0.18 & 0.11 & 0.14 & 80 \\
\hline ID_3_886_0 & 36 & 52.92 & 12 & 03.11 & $\mathrm{E}$ & $\mathrm{E}$ & 2 & $25.21(.18)$ & 0.14 & 0.16 & 0.21 & 8 \\
\hline ID_2_726_1 & 36 & 53.12 & 13 & 46.25 & $\mathrm{E}$ & - & 1 & $26.75(.19)$ & 0.11 & 0.23 & 0.18 & 35 \\
\hline ID_2_635_0 & 36 & 53.15 & 13 & 31.66 & $\mathrm{E}$ & - & 1 & $25.99(.15)$ & 0.17 & 0.24 & 0.24 & 29 \\
\hline ID_2_591_2 & 36 & 53.18 & 13 & 22.75 & $\mathrm{E}$ & - & 2 & $25.05(.15)$ & 0.18 & 0.31 & 0.30 & 10 \\
\hline ID_3_670_1 & 36 & 53.26 & 12 & 22.74 & $\mathrm{E}$ & $\mathrm{E}$ & 2 & $25.73(.21)$ & 0.14 & 0.28 & 0.26 & 20 \\
\hline ID_2_643_0 & 36 & 53.42 & 13 & 29.52 & $\mathrm{~m}$ & $\mathrm{E}+\mathrm{E}$ & 3 & 25.03(.17) & 0.22 & 0.65 & 0.62 & 5 \\
\hline ID_2_973_2 & 36 & 54.50 & 14 & 08.16 & $\mathrm{E}$ & - & 2 & $25.88(.18)$ & 0.18 & 0.23 & 0.19 & 10 \\
\hline ID_3_118_1 & 36 & 54.73 & 13 & 14.73 & $\mathrm{E}$ & $\mathrm{E} / *$ & 1 & $24.59(.17)$ & 0.14 & 0.08 & 0.09 & 10 \\
\hline ID_2_898_0 & 36 & 54.78 & 13 & 50.74 & $\mathrm{~m}$ & Sa & 3 & $25.14(.20)$ & 0.27 & 0.49 & 0.50 & 3 \\
\hline ID_3_743_0 & 36 & 54.95 & 12 & 21.44 & $\mathrm{E}$ & - & 2 & $25.61(.20)$ & 0.19 & 0.11 & 0.14 & 93 \\
\hline ID_3_266_0 & 36 & 55.16 & 13 & 03.60 & $\mathrm{E}$ & $\mathrm{E}$ & 1 & $25.19(.19)$ & 0.22 & 0.30 & 0.31 & 3 \\
\hline ID_3_180_1 & 36 & 55.46 & 13 & 11.19 & $\mathrm{E} / \mathrm{S} 0$ & $\mathrm{E}$ & 1 & $23.94(.21)$ & 0.51 & 0.18 & 0.19 & 70 \\
\hline ID_2_966_0 & 36 & 55.77 & 13 & 48.78 & $\mathrm{E}$ & $\mathrm{E}$ & 2 & 25.83(.19) & 0.18 & 0.24 & 0.24 & 40 \\
\hline ID_3_904_0 & 36 & 55.95 & 12 & 10.72 & $\mathrm{E}$ & $\mathrm{E}$ & 1 & 25.11(.13) & 0.17 & 0.08 & 0.14 & 70 \\
\hline ID_3_815_1 & 36 & 56.65 & 12 & 20.12 & $\mathrm{E} / \mathrm{S} 0$ & $\mathrm{E}$ & 1 & $24.29(.20)$ & 0.41 & 0.21 & 0.22 & 11 \\
\hline ID_3_355_0 & 36 & 56.92 & 13 & 01.56 & E & $\mathrm{E}$ & 1 & $24.16(.20)$ & 0.39 & 0.04 & 0.08 & 5 \\
\hline ID_3_726_0 & 36 & 58.53 & 12 & 33.59 & $\mathrm{E}$ & - & 2 & $26.15(.18)$ & 0.18 & 0.29 & 0.28 & 12 \\
\hline ID_3_363_1 & 36 & 58.57 & 13 & 05.47 & $\mathrm{E}$ & - & 1 & $26.23(.20)$ & 0.11 & 0.05 & 0.04 & 5 \\
\hline ID_3_813_0 & 36 & 59.24 & 12 & 27.29 & $\mathrm{E}$ & - & 1 & $26.02(.18)$ & 0.17 & 0.04 & 0.08 & 135 \\
\hline ID_3_748_0 & 36 & 59.98 & 12 & 35.95 & $\mathrm{E}$ & - & 1 & $26.23(.17)$ & 0.15 & 0.23 & 0.20 & 16 \\
\hline ID_3_888_1 & 37 & 00.52 & 12 & 25.81 & $\mathrm{E}$ & Sap & 1 & $25.57(.18)$ & 0.17 & 0.12 & 0.18 & 42 \\
\hline ID_3_790_1 & 37 & 00.56 & 12 & 34.60 & $\mathrm{E}$ & - & 1 & $22.60(.14)$ & 0.40 & 0.10 & 0.13 & 20 \\
\hline
\end{tabular}

residuals as a function of the semi-major axes of the fitted isophote.

The most important contribution to the uncertainties in the outer profiles of nearby elliptical galaxies from CCD data is usually given by the possible errors in the estimate of the background. In particular, an underestimation (overestimation) of the average background level leads to a systematic distortion upwards (downwards) of the outer luminosity profiles and then to an overestimation (underestimation) of the total apparent luminosity. In our case this kind of uncertainty is not the dominant one since the average value of the background is very carefully estimated (well outside the faint galaxy halos) by the above mentioned "local refinement". A more serious problem is represented by the possible systematicity of the background residuals. Fasano \& Bonoli (1990) analyzed the influence of an artificially tilted background on the luminosity and geometrical profiles, giving a set of formulae to estimate error bars of surface brightness, ellipticity and position angle of each isophote, mainly depending on the relative background variation $(0.5 \%$ in our case; see previous subsection) and on the considered isophotal level. Error bars of our profiles were computed according to these formulae, with an additional term (which could be relevant for the outer isophotes) accounting for the number of points belonging to each isophote. This term turns out to be usually negligible for ground-based surface photometries, where the small signal to noise ratio of the outermost regions is largely compensated for the great number of points defining the isophotes. In our case, the incredible deepness and resolution of the images allowed us to perform the detailed surface photometry of galaxies in which even the outermost isophotes are defined by a relatively small number of points.

\section{Results}

The profiles of surface brightness $(\mu)$, ellipticity $(\varepsilon)$, position angle $(\theta)$ and coefficient of the Fourier analysis of the residuals $\left(c_{4}\right)$, as a funtion of the semi-major axis $a$ 
in arcseconds ( $a^{1 / 4}$ scale), are available on the online version of the journal (Fig. 1), together with the proper error bars (see previus section). In the same figures we report for comparison the $P S F$ profile as a dotted line.

\subsection{Statistical properties of profiles}

\subsubsection{Luminosity profiles}

We divided our galaxy sample in 3 different classes, according to the luminosity profiles:

- most of galaxies (55) have luminosity profiles following reasonably well the de Vaucouleurs law up to the innermost isophote not significantly affected by the $P S F$ (hereafter "Normal" class);

- a relevant fraction of galaxies (36), even following the de Vaucouleurs law in the main body light distribution, exhibit in the inner regions a substantial flattening of the luminosity profiles, not attributable to the $P S F$ (hereafter "Flat" class);

- finally, the isophotal analysis allowed us to detect 8 galaxies showing complex inner structures (most of them are multi-nucleus objects), but still obeying the de Vaucouleurs law in the outer profiles (hereafter "Merger" class).

In Col. (6) of Table 1 the luminosity profile class of each galaxy is reported and the three classes are indicated with 1,2 and 3 , respectively. The same convention is used in Table 2.

Concerning the "Flat" class, it is worth stressing that the observed inward flattening of the luminosity profiles with respect to the de Vaucouleurs law cannot be interpreted as due to the presence of some core-like structure. Actually, in all galaxies of this class for which the redshift has been measured ( 6 objects), the linear size of the involved regions turns out to be much greater than the typical core size $\left(10^{2} \mathrm{pcs}\right)$. For instance, at $z \sim 1,10^{2} \mathrm{pcs}$ correspond to $\sim 00^{\prime \prime} 01$, with small differences in the range $0.5 \leq z \leq 3$. On the other hand, galaxies with luminosity profile flattening confined inside $\sim 0^{\prime \prime} .1$ have been included by default in the "Normal" class. Moreover, by comparing the fraction of HDF ellipticals belonging to the "Flat" class with that obtained in a similar way from the sample of local ellipticals provided by Djorgovski (1985), we found a relevant difference in favour of the HDF sample $(\sim 36 \%$ vs. $\sim 7 \%$ ). We will see in a forthcoming paper (Fasano et al. 1998) that the different classes of luminosity profiles are often associated with different physical properties of the galaxies (i.e. colors and sizes).

Finally, we mention that the luminosity profile of the galaxy $I D \_2 \_251 \_0$ strongly suggests the presence of a nuclear point source. This galaxy also belongs to the lists of radio and ISO sources in the HDF (Fomalont et al. 1997; Mann et al. 1997).

\subsubsection{Ellipticity profiles}

The shape of the ellipticity profiles appears to be correlated with the above mentioned luminosity profile classes. Actually, the galaxies belonging to the "Normal" class show increasing or almost constant ellipticity profiles (see Fig. 1). This is the most common behaviour in nearby early-type galaxies (see Bettoni et al. 1996). On the other hand, 25 out of the 44 galaxies belonging to the "Flat" class or to the "Merger" class show strongly decreasing ellipticity profiles (see Fig. 1). This is an unusual behaviour in the local samples of early-type galaxy (Bettoni et al. 1996). Table 2 reports some statistical data on the shapes of the ellipticity profiles.

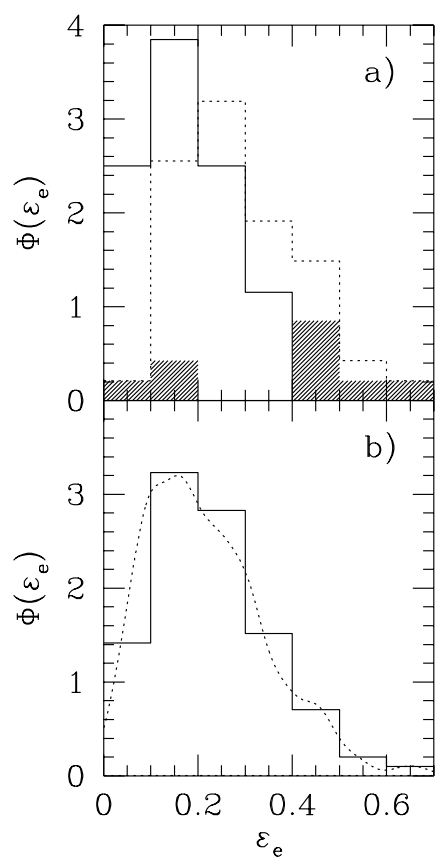

Fig. 2. a) effective ellipticity distribution of the "Normal" galaxies (full line histogram) campared with that of "Flat+Merger" (dotted line histogram). The shaded histogram refer to the "Merger" class alone. b) effective ellipticity distribution of the total sample (full line histogram) compared with that of "local" sample from the literature (Fasano \& Vio 1991)

This peculiarity of the ellipticity profiles is likely to reflect on the ellipticity distribution (computed at the effective radius, see Col. 9 of Table 1) of galaxies in our sample. Figure 2a shows that the ellipticity distribution of the "Normal" class looks remarkably different from that relative to the other two classes, the last ones being shifted towards flatter configurations. The KolmogorovSmirnov test confirms this difference at high significance level $(98.5 \%)$. Nevertheless, the ellipticity distribution of 
the whole sample (Fig. 2b) seems to be in fair agreement with that relative to nearby galaxy samples (see Fasano \& Vio 1991). It is worth stressing that the comparison with the local samples is not invalidated by the fact that the selection criteria of our sample extend to the limit of recognition between stars and galaxies. In fact the error bars shown in the ellipticity profiles take into account the influence of the PSF (see Sect. 3.2).

\subsubsection{Isophotal shape and twisting}

In Table 2 we report some statistics on the shape of the isophotes ("disky" for $c_{4}>0$; "boxy" for $c_{4}<0$ ) in our galaxy sample for the different luminosity profile classes. There is a weak indication that the fraction of boxy galaxies increases from the "Normal" to the "Flat" and "Merger" classes.

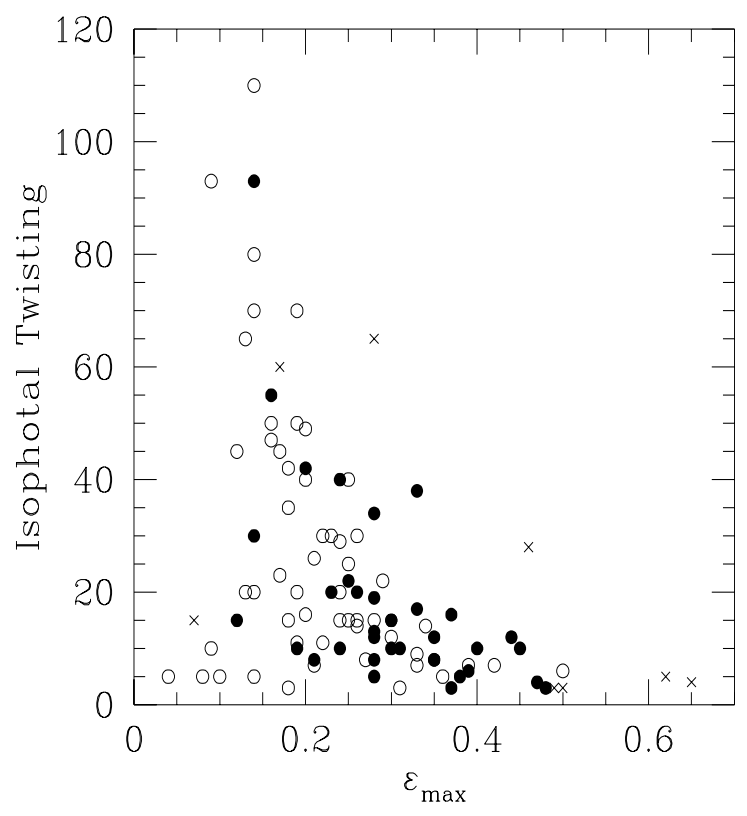

Fig. 3. Maximum ellipticity vs. isophotal twisting (open circles: "Normal" class; full circles: "Flat" class; crosses: "Merger" class)

Concerning the position angle profiles, although the uncertainties involved in measuring the position angle of outer isophotes are relevant for HDF ellipticals, we estimate that the amount of isophotal twisting in our sample is larger (on average) than that found in local galaxy samples (see Fasano \& Bonoli 1989). This fact may be explained as a consequence of the high fraction of morphologically perturbed objects in the HDF.

Figure 3 shows the distribution of $H D F$ early-type galaxies in the "maximum ellipticity - twisting" plane, which, even being qualitatively similar to that of local samples (see Galletta 1980), shows an higher fraction of significantly twisted objects. The maximum ellipticity $\varepsilon_{\max }$ and the total isophotal twisting (in degrees) are reported in Cols. (10) and (11) of Table 1, respectively.

Table 2. Statistical properties of profiles

\begin{tabular}{|c|c|c|c|c|}
\hline Class & 1 & 2 & 3 & All \\
\hline \multicolumn{5}{|c|}{ average ellipticities } \\
\hline$<\varepsilon_{\mathrm{e}}>$ & 0.17 & 0.28 & 0.38 & 0.23 \\
\hline$<\varepsilon_{\max }>$ & 0.21 & 0.30 & 0.40 & 0.26 \\
\hline \multicolumn{5}{|c|}{$\begin{array}{l}\text { ellipticity profiles (\%) } \\
\end{array}$} \\
\hline$\overline{\varepsilon \nearrow}$ & 36 & 14 & 0 & 25 \\
\hline$\varepsilon \sim$ & 58 & 33 & 22 & 46 \\
\hline$\varepsilon \searrow$ & 6 & 53 & 75 & 29 \\
\hline \multicolumn{5}{|c|}{ isophotal shape (\%) } \\
\hline disky & 33 & 25 & 11 & 28 \\
\hline elliptical & 14 & 11 & 0 & 12 \\
\hline boxy & 22 & 33 & 50 & 29 \\
\hline irregular & 31 & 31 & 33 & 31 \\
\hline \#gal. & 55 & 36 & 8 & 99 \\
\hline
\end{tabular}

\subsection{Extraction of the global parameters}

In order to derive total magnitudes and half-light radii of the galaxies, it is convenient to use some analytical representation of the luminosity profiles. This allows a suitable smoothing of each profile and provides an easy way to extrapolate it. For obvious reasons it is also convenient to operate on the "equivalent" luminosity profiles, that is to multiply the semi-major axis $(a)$ by the factor $\sqrt{1-\varepsilon(a)}$.

The most common technique to get suitably smooth representations of any observed function is the bicubicspline interpolation. In the case of luminosity profiles of elliptical galaxies, a nice representation is also given by the Sersic function (1968, see also Ciotti 1991). However, we preferred to represent the equivalent luminosity profiles by means of sums of gaussian functions whose peak intensities regularly decrease at increasing the standard deviations (multi-gaussian expansion technique). In a forthcoming paper (Fasano et al. 1998) we will see that this representation is useful to perform the deconvolution of the luminosity profiles (Bendinelli 1991; Emsellem et al. 1994). Here we wish only to mention that, in our particular case, this method gives usually a better representation with respect to the bicubic-spline method, especially in the inner part of luminosity profiles.

The multi-gaussian representation was also used to extrapolate the profiles. In general, it was forced to follow the de Vaucouleurs law down to very faint values of $\mu$. However, if the galaxy size is comparable with the PSF size (very steep profiles), it is necessary to impose that 


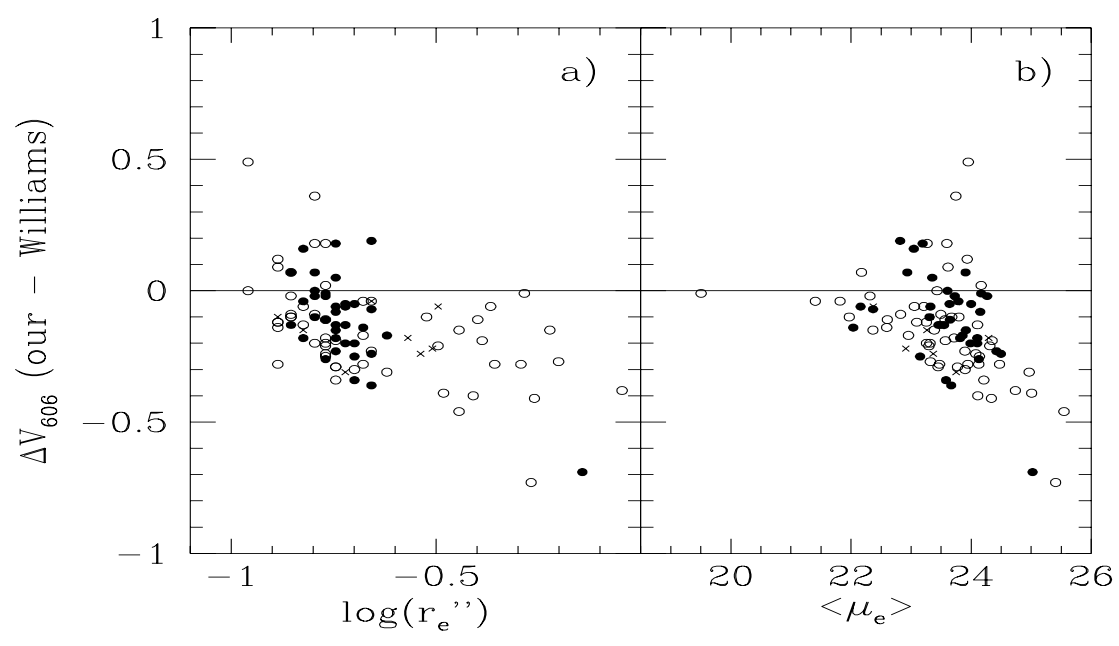

Fig. 4. Difference between our total magnitudes and the magnitudes given by Williams et al. (1996) as a function of the effective radius a) and of the average surface brightness $\mathbf{b}$ ). The meaning of the symbols is the same as in Fig. 3

the multi-gaussian extrapolation of the outer galaxy profile does not fall below the very extended wings of the $H S T-P S F$ itself. To this end we forced the extrapolation of the luminosity profiles of very small galaxies to converge smoothly towards the $P S F$ profile at large radii. Luminosity profiles with an effective radius larger than three times the $F W H M$ were extrapolated simply by a de Vaucouleurs' law.

A special warning is needed when computing the total magnitude of galaxies belonging to the above defined "Merger" class, since their complex inner structures make undefined (or unreliable) the inner part of luminosity profiles. In these cases the flux inside the innermost reliable ellipse was directly mesured on the frame and was considered as an additional contribution to the integral of the luminosity profile, computed from that ellipse and extrapolated by a de Vaucouleurs law. The total magnitudes in the $V_{606}$ band (STMAG system) and the corresponding equivalent effective (half-light) radii $r_{\mathrm{e}}$ are reported in the Cols. (7) and (8) of Table 1, respectively. The quantities in brackets close to the Col. (7) represent the surplus magnitudes $\Delta_{m}$ due to the extrapolation procedure. They give an indication of the quality of the total magnitude estimates. Adding these quantities to $V_{606}^{\text {Tot }}$ one obtain, for each galaxy, the magnitude before extrapolation.

In Fig. 4 we compare the $V_{606}-S T M A G$ total magnitudes from our detailed surface photometry with the automated FOCAS magnitudes given by Williams et al. (1996), corrected for the average offset of 0.2 between the $A B M A G$ and the $S T M A G$ systems. There are two galaxies (ID_2_726_1 and $\left.I D \_4 \_289 \_0\right)$ for which the flux has been probably overestimated by $F O C A S$, due to the presence of very close companions. Apart from these cases, the total fluxes computed with our procedure tend to be systematically greater than those obtained with the automated photometry. Moreover, the difference increases at increasing both the average surface brightness of the galaxies and (weakly) their angular size. This fact is not surprising and it is likely to indicate that the automated photometry tends to underestimate the halos of the ellipticals. To this concern, it is also worth noticing that the differences $\Delta V_{\text {mag }}$ in Fig. 4 turn out to be roughly proportional to the previously mentioned quantities $\Delta_{m}$.

\section{References}

Bendinelli O., 1991, ApJ 366, 599

Bertin E., Arnouts S., 1996, A\&AS 117, 393

Bettoni D., Fasano G., Kjaergaard P., Moles M., 1996, Second Stromlo Symposium: The Nature of Elliptical Galaxies, ASP Conf. Ser. 116, 71

Chiosi C., Bressan A., Portinari L., Tantalo R., 1997, A\&A, babbage astro-ph/9708123 (submitted)

Ciotti L., 1991, A\&A 249, 99

Couch W.J., 1996, http://ecf.hq.eso.org/hdf/catalogs/

Djorgovski S., 1985, Ph.D. Thesis, University of California, Berkeley

Djorgovski S., Davis M., 1987, ApJ 313, 59

Dressler A., Lynden-Bell D., Burstein D., et al., 1987, ApJ 313, 42

Ellis R.S., 1997, Proc. of the STScI May Symposium: The Hubble Deep Field, babbage astro-ph/9708075

Emsellem E., Monnet G., Bacon R., 1994, A\&A 285, 723

Fasano G., 1990, internal report of the Padova Astronomical Observatory

Fasano G., Bonoli C., 1989, A\&AS 79, 291

Fasano G., Bonoli C., 1990, A\&A 234, 89

Fasano G., Vio R., 1991, MNRAS 249, 208

Fasano G., Cristiani S., Arnouts S., Filippi M., 1998, AJ (in press)

Filippi M., Fasano G., 1998 (in preparation) 
Fomalont E.B., Kellermann K.I., Richards E.A., Windhorst R.A., Partridge R.B., 1997, ApJ 475, L5

Galletta G., 1980, A\&A 181, 179

Giavalisco M., Steidel C.C., Macchetto F.D., 1996, ApJ 470, 189

Kauffmann G., Charlot S., 1997, MNRAS 294, 705

Jenkins A., Frenk C.S., Pearce F.R., et al., 1997, ApJ, babbage astro-ph/9709010 (submitted)

Lowenthal J.D., Koo D,C., Guzman R., 1997, ApJ 481, L673

Madau P., Pozzetti L., Dickinson M., 1997, ApJ, babbage astro-ph/9708220 (submitted)

Mann B., et al., (The ISO-HDF Consortium) 1997, MNRAS (submitted)

Oemler A.Jr., Dressler A., Butcher H.R., 1997, ApJ 474, 561

Pettini M., Steidel C.C., Dickinson M., 1997, Proc. of the meeting: The Ultraviolet Universe at Low and High Redshift, Waller W. (ed.) babbage astro-ph/9707200 Woodbury: AIP Press
Sersic J.L., 1968, "Atlas de galaxias australes", Observatorio Astronomico de Cordoba, Argentina

Shade D., Carlberg R.G., Yee H.K.C., Lopez-Cruz O., Ellingson E., 1996, ApJ 464, L63

Shade D., Barrientos L.F., Lopez-Cruz O., 1997, ApJ 477, L20

Statler T., 1996 (private communication)

Tantalo R., Chiosi C., Bressan A., Fagotto F., 1996, A\&A 311, 361

Tully R.B., Fisher J.R.T., 1977, A\&A 54, 661

van den Bergh S., Abraham R.G., Ellis R.S., et al., 1996, AJ 112,359

van Dokkum P.G., Franx M., 1996, MNRAS 281, 985

White S.D.M., 1997, Proc. of the 3rd ESO-VLT Workshop: Galaxy Scaling Relations: Origins, Evolution and Applications, p. 3

Williams R.E., Blacker B., Dickinson M., et al., 1996, AJ 112, 1335 